\title{
Cooperative continuum robots: Enhancing individual continuum arms by reconfiguring into a parallel manipulator
}

\author{
Matteo Russo, Natthapol Sriratanasak, Weiming Ba, \\ Xin Dong, Abdelkhalick Mohammad, and Dragos Axinte
}

\begin{abstract}
Continuum robots are able of in-situ inspection tasks in cluttered environments and narrow passages, where conventional robots and human operators cannot intervene. However, such intervention often requires the robot to interact with the environment, and the low stiffness and payload of continuum robots limits their intervention capabilities. In this paper, we propose a paradigm shift from individual to multiple continuum robots, which can reach the target environment from different paths and then physically connect, reconfiguring into a parallel architecture to enhance precision, stiffness, and payload. The main challenges in modelling and controlling cooperative continuum robots are outlined, and an experimental comparison between individual and cooperating continuum robots that connect through a novel shape-memory-alloy-based clutch highlights the advantages of the proposed technology.
\end{abstract}

Index Terms-Cooperating Robots; Mechanism Design; Continuum Robots; Compliant Mechanisms; Parallel Robots.

\section{INTRODUCTION}

The inspection and maintenance of capital-intensive industrial infrastructures such as aeroengines, nuclear facilities, and telecommunication networks are critical tasks to achieve safety and reliability. Unfortunately, these operations are not only expensive and time-consuming, but also often needed in challenging scenarios where human operators cannot intervene due to hazards such as radioactivity or extreme temperatures. Thus, even a routine inspection to a single component can lead to a long downtime of the entire infrastructure. While an autonomous or teleoperated robotic system could perform these key operations in-situ (on site, without infrastructure shutdown or disassembly), conventional industrial robots cannot navigate in environments such as gas turbines and pipes, characterized by passages that are narrow ( $<20 \mathrm{~mm}$ ), long (i.e., up to several meters), and characterized by many sharp turns and obstacles (e.g., turbine blades).

In the last decade, continuum robots have been identified as the most promising solution for these applications [1]. These slender robots are tailored to navigate through narrow spaces and complex geometries, thanks to a small outer diameter (6-12 $\mathrm{mm}$ in industrial applications [2], and even smaller in surgical robots [3]), a long reach, and a large number of degrees of freedom (DoFs) (e.g., length of $1 \mathrm{~m}$ and 17 DoFs

Manuscript received: August 18, 2021; Revised November 8, 2021; Accepted December 23, 2021.

This paper was recommended for publication by Editor Clement Gosselin upon evaluation of the Associate Editor and Reviewers' comments. This work was supported by UK EPSRC project Robotics and Artificial Intelligence in Nuclear Plus (RAIN+) under grant EP/W001128/1. in [4]). However, their low stiffness results in a low precision of tip positioning and a limited payload [5-7]. Currently, even if continuum robot operation has been demonstrated in either non-contact [8] or short reach [9] scenarios, no hardware solution offers both a long reach and the capability to physically interact with the environment in precision tasks, as summarized in the scheme in Fig. 1.

As a fundamentally novel solution, we propose the cooperation of multiple continuum robots to overcome these issues. Some of the advantages of two continuum robots supporting each other on a task have been already outlined in [10-11], where a flame-spraying continuum robot is monitored by a second continuum robot in a coating operation within an aeroengine. Even though the control of these robots is enhanced by the cooperation, their mechanical performance (i.e., stiffness/payload) is not improved. Conversely, two or more continuum robot can navigate individually and independently to meet in the target workspace, where they can physically interface to reconfigure into a parallel robot with increased stiffness and payload. The use of continuum structures for the limb of parallel robots has been studied in several recent research works [12-13], outlining their advantages over conventional parallel manipulators. However, existing parallel continuum robots (e.g. [14-17]) cannot be deployed in many challenging scenarios because of their shape factor and short reach. Some parallel continuum robots can adapt their geometry to the task: the base position and orientation of the prototype in [18] can be manually reconfigured to comply with environmental constraints, while the steerable needle in [19] is guided by parallel "snare" limbs to improve its motion control. These systems, however, cannot enter the typical work areas for industrial continuum robots, such as turbines and pipes, which all require a long reach with a narrow access port. On the other hand, the capability to reconfigure individual continuum arms, which can navigate independently to secluded workspaces, into a parallel mechanism could enable the usage of these structures in aeroengines and similar challenging environments.

Thus, this manuscript introduces a new concept of reconfigurable continuum robots with experimental examples. The proposed robots are able of independent individual operation and can (optionally) physically connect to each other

M. Russo, N. Sriratanasak, W. Ba, X. Dong, A. Mohammad, and D. Axinte are with the Rolls-Royce UTC in Manufacturing and On-Wing Technology, Faculty of Engineering, University of Nottingham, Nottingham NG8 1BB, United Kingdom (e-mail: \{matteo.russo; natthapol.sriratanasak; weiming.ba; xin.dong; abd.mohammad; dragos.axinte\}@nottingham.ac.uk)

Digital Object Identifier (DOI): see top of this page. 
to cooperate as a parallel manipulator for improved mechanical performance. In this way, the proposed concept can benefit from the advantages of a parallel architecture without sacrificing reach or navigation capabilities like previous parallel continuum robots. While there is a rich literature of conventional robots cooperating for improved performance, the hyper-redundant architecture and added complexity of continuum robots pose an additional challenge that has never been addressed before. The main challenges and benefits of the cooperation between continuum robots are thus discussed in Section II. Section III presents an exemplary design solution for the reconfiguration of individual continuum robots into a parallel manipulator, based on a new Shape-Memory Alloy (SMA) clutch design. In Section IV, the proposed clutch is installed on two continuum robots, whose cooperation is characterized with experimental tests. Section $\mathrm{V}$ discussed the results of the experimental tests by comparing them to the performance of the individual continuum robots. Section VI includes final remarks and suggests applications and future developments for cooperative continuum robots.
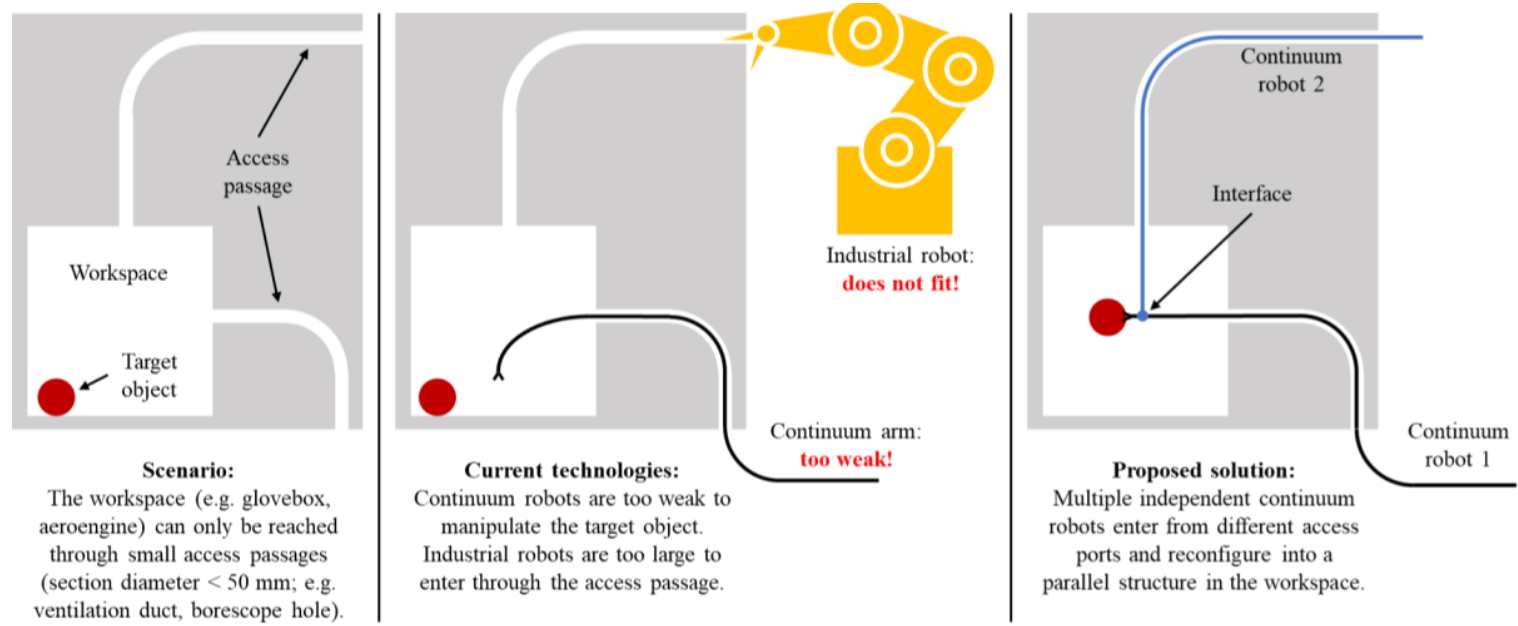

Figure 1. A graphical representation of the research challenge of operating in cluttered and hard-to-reach environments and the proposed solution with reconfigurable continuum robots cooperating, which overcome the intrinsic limits of individual continuum robots while still being able to access areas precluded to conventional industrial manipulators.

\section{CONTINUUM ROBOT COOPERATION}

In this section, we analyze the nature of the cooperation between multiple continuum robots. Cooperative tasks can be classified by the mechanical interaction between robots, identifying the following categories:

- Disjunctive cooperation, when multiple robots work toward a common goal without physical contact.

- Conjunctive cooperation, when multiple robots operate by physically interfacing with each other.

An example of disjunctive cooperation can be found in the FLARE project [10], in which two continuum robots perform coating repair without any physical interaction: one of the robots carries a flame, while the other generates the spark to ignite the flame and carries a camera to monitor the process. In this paper, we focus on the fundamentally new conjunctive cooperation instead, where, by joining their tips and thus behaving as a parallel manipulator, multiple continuum robots can increase their stiffness and accuracy. Both disjunctive and conjunctive tasks are discussed with their characteristics and potential applications in this section, in order to outline the main features of cooperative continuum robots.

\section{A. Disjunctive cooperation}

Given the complex environments where continuum robots operate, obtaining external feedback is often difficult or impossible. While robot proprioception is possible, the most advanced shape sensors such as fiber Bragg grating (FBG) still incur in small errors (1 to 2 deg over $11 \mathrm{~mm}$ [20]), which are significantly amplified in the $1 \mathrm{~m}$ or longer backbones for longreach industrial applications [4]. Thus, one of the main advantages of disjunctive cooperation is the possibility to measure the position of a continuum robot from the point of view of the other robots in the system. As illustrated in the example in Fig. 2a, two continuum robots can estimate the pose of their end-effectors $\mathrm{H}_{1}$ and $\mathrm{H}_{2}$ by measuring the relative distance $\mathrm{h}_{21}$ between them and/or by evaluating the relative position of the other robot to the environment. Especially when operating in environments where the geometry is known, such as aeroengines or nuclear vessels, this reciprocal feedback can enable closed-loop control to improve both navigation and operational precision.

\section{B. Conjunctive cooperation}

The intrinsically low stiffness of continuum robot backbones significantly hinders the performance of the system in terms of payload and precision (because of robot sagging) [5], especially when an operation requires a fully suspended system that cannot "lean" on any obstacle. In absence of this requirement, a vine-like motion that employs environmental features to support the backbone has been proposed by several research groups [21-22] as a solution to reduce undesired deformations. However, this strategy is not always possible, as the environment might lack suitable features (e.g., in a vertical pipe), or the robot might not be able to exploit them (e.g., sharp edges). These remaining scenarios are where conjunctive cooperation is required the most, since it provides a way to enhance the mechanical performance of the system without relying on any external or environmental resource.

The main advantage of conjunctive cooperation is an increase in stiffness and payload of the combined system [23]. This can enable operations where force or pressure are critical. 
An example of a combined system is shown in the example in Fig. 2b, where a continuum robot (1) is supported by another (2) through an interface that transmits wrenches $\left(R_{21}\right)$. Such a system behaves as a parallel manipulator with the continuum robots acting as parallel limbs, with the consequent advantages on accuracy and static performance [23-24]. From a control point of view, the system is redundant, and it can be controlled by solving the redundancy with a release in the tension of some driving cables, so that some active DoFs become passive, or by using the redundant DoFs to "stiffen" the system.

Further advantages of conjunctive cooperation include the potential to better distribute tools and sensor in the endeffector(s). For example, by designing an interface that can transmit current, one of the robots could be used as a power line to a supply, freeing up the extremely limited space in the inner channels of the other continuum robots in the system.

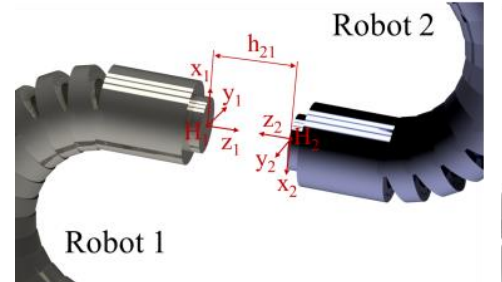

a.

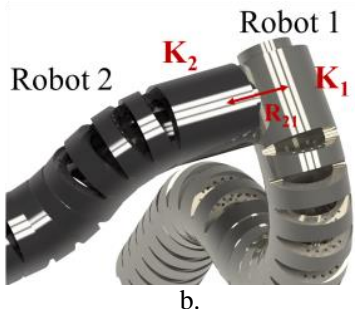

Figure 2. Cooperating continuum robots: a. Disjunctive cooperation between robots that observe each other to increase accuracy; b. Conjunctive cooperation between robots that connect to increase payload and stiffness.

\section{Reconfigurable continuum robots in cooperative tasks}

While we can classify a given cooperative task as conjunctive or disjunctive, a similar distinction does not apply to the continuum robots themselves, as the proposed reconfigurability concept implies the capability of the individual robots in the system to operate individually and independently, albeit with reduced performance. Therefore, even if they are designed for conjunctive cooperation, the single continuum robots of the system could still be able to perform disjunctive and/or independent tasks. Thus, we define different types of continuum robots in a cooperative system according to their reliance on others as follows:

- Independent robots can successfully navigate and operate alone but enjoy improved performance when cooperating with others.

- Partially dependent robots are characterized by limited functionality when operating alone. For example, one of the robots in [11] carries a camera and can be used for inspection independently. However, it cannot perform the coating repair (no gas supply).

- Fully dependent robots cannot operate alone. For example, the spraying robot in [10-11] relies on the other to ignite the flame, and the "snares" in [18] have no capabilities on their own.

The choice among these different kinds of robots depends on the scenario, but, overall, cooperation enables a whole new range of applications for continuum robots. In the next sections of this manuscript, a specific application of conjunctive cooperation is reported as an example of the advantages of reconfigurable continuum robots.

\section{A MECHANICAL SOLUTION FOR COOPERATION}

The key element of conjunctive cooperation is the interface mechanism between the continuum robots. Its requirements can be summarized in the following points:

- Enable robot connection: When the robots are next to each other in a desired relative position to each other, the interface must join the robots together.

- Enable robot separation: At the end of the conjunctive operation, the robots must continue operation individually or be retracted as such. The interface must provide a release mechanism for the robots to separate without any external intervention.

- Allow robot navigation: The interface mechanism should not hinder robot navigation and operation. Thus, it should be fully contained in a cylinder with the same external diameter of the robot, with an inner channel to deliver an end-effector (if needed) and a rigid length limited by environmental constraints.

- Provide wrench transmission: By being the physical link that joins the robots together, the interface mechanism characterizes the performance of the whole conjunctive system. Therefore, any wrench that is transmitted through it must not cause the separation of the parts of the interface mechanism.

In this section, a miniature gripper based on SMA is proposed as an example solution for the interface between cooperative continuum robots. Its conceptual design is described, and a prototype is manufactured to test the cooperation between continuum robots.

\section{A. Conceptual design of a SMA-based interface mechanism}

To enable the physical interaction between continuum robots, we propose a grasping mechanism with a spring mechanism for the connection between two robots and a SMAbased release mechanism. Since the interface mechanism is close to the end-effector of the robot, an intrinsically actuated mechanism enables a more efficient force transmission [3]. Given the size constraint, only technologies that can be miniaturized can be selected, such as SMAs, dielectric or piezoelectric actuators, pneumatics, and electromagnets. Among them, electromagnets provide a high grasping force but are characterized by bulky shapes and a significant weight, which would cause an undesired deflection on the slender continuum robot; dielectric and piezoelectric actuators provide a limited motion, which would make the engagement motion challenging by requiring a high positioning accuracy; SMAs have ultimately been selected over pneumatics as they occupy less space in the inner channel of the robot (electric wires rather than fluid hose). However, any equivalent actuation technology could be used to validate the cooperation concept.

The functioning of the proposed mechanism is illustrated in the conceptual diagram in Fig. 3a. One of the two robots (2) has a purely passive role, with a cross-section shape designed to be grasped by the clutch. The other continuum robot (1) is equipped with a compliant gripper based on a flexible hinge and a SMA wire. When the passive robot pushes onto the clutch, the flexible hinge, which is designed to have a low stiffness, bends, opening the clutch. Once the coupling 
element of the passive robot is fully within the clutch, the elastic force of the flexible hinge restores it back to its original configuration, grasping the passive robot with a shape coupling (rather than by friction only) and locking the two robots together. To release the passive robot, the SMA wire is heated and straightens by activating its shape memory, thus opening the clutch for an easy disengagement of the robots.

\section{B. Prototype of a SMA-based interface mechanism}

A model of the proposed concept has been designed as shown in Fig. 3b to interface with the RAIN-Snake continuum robot ([4], with a $20 \mathrm{~mm}$ outer diameter) as robot 1 , and the FLARE continuum robot ([8], with a $12 \mathrm{~mm}$ outer diameter) as robot 2. The four bodies in Fig. $3 \mathrm{~b}$ have been manufactured in High Temp Resin with a Formlabs Form 3 SLA 3D printer and installed onto the RAIN-Snake [4] and FLARE [8] continuum robots as shown in Fig. 4. The shape-memory alloy is a MuscleWires Flexinol LT with a diameter of $250 \mu \mathrm{m}$ and an activation temperature of $70^{\circ} \mathrm{C}$.

The two robots can engage by either pushing the tip of robot 2 onto robot 1 , which opens the clutch by deforming the flexible hinge until the grasping element "snaps" onto it (similarly to a seat belt mechanism; opening load: $0.2 \mathrm{~N}$ ), or by opening the clutch with the SMA before positioning robot 2 onto robot 1 and then closing the clutch again. Conversely, robot 2 can be only released by heating the SMA to open the grasping element, since once the clutch is closed, the shape fit prevents an accidental separation of the two continuum robots. A radial load of up to $10 \mathrm{~N}$ has been applied to the connected mechanism to test its grasping force and no undesired behavior has been observed. Higher loads can cause a mechanical failure of the system due to the limited strength of the mechanism, which is made of resin and includes thin features (thickness $<1 \mathrm{~mm}$ ) that can be damaged by an excessive load.
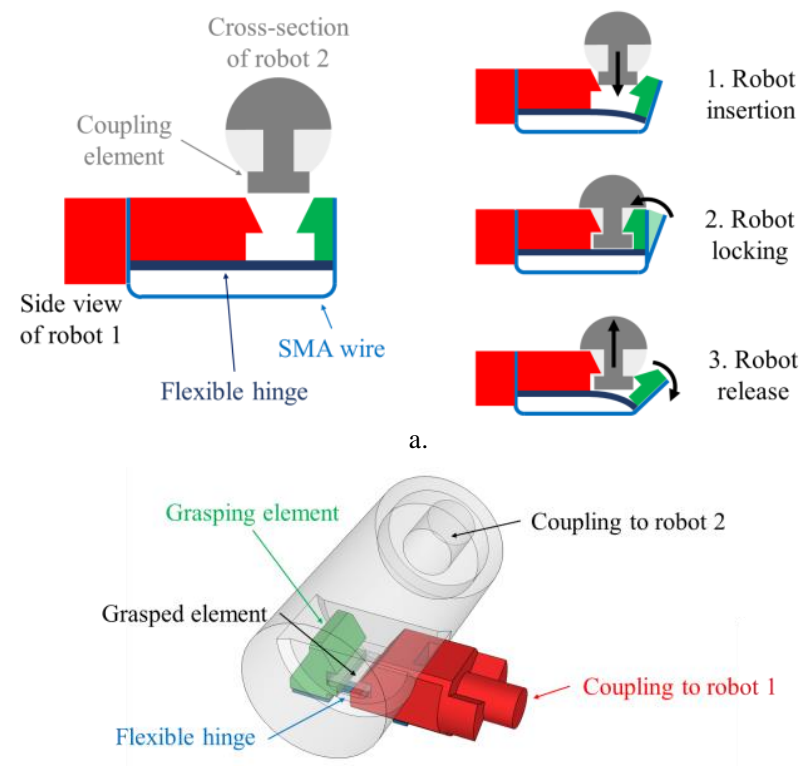

b.

Figure 3. The proposed SMA-based clutch mechanism: a. Operation in three main steps, i.e. robot insertion (the flexible hinge deflects to open the clutch), robot locking (the hinge springs back closed once the coupling element is fully grasped), and robot release (the SMA opens the clutch when heated); b. A 3D model of an example of SMA-based interface mechanism with main bodies, i.e. a coupling for robot 1 (in red), a flexible hinge (in blue), the grasping element (in green), and a coupling for robot 2 (in grey).

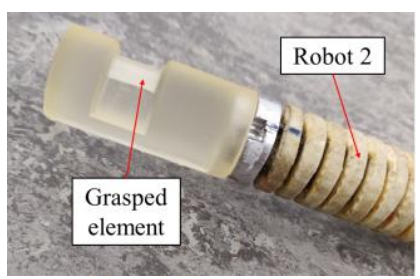

a.
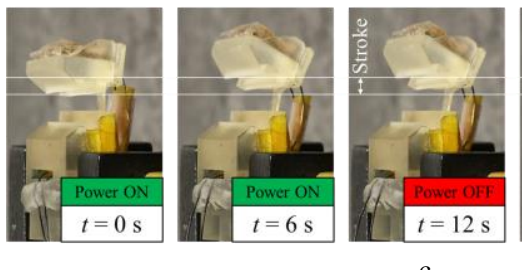

c.

Figure 4. An example prototype of the proposed SMA-based interface mechanism: a. The grasped element installed at the tip of the RAIN-Snake continuum robot [4]; b. The grasping mechanism installed at the tip of the FLARE continuum robot [8]; c. Snapshot of the clutch activation and deactivation, applying a current for the first $12 \mathrm{~s}$ only.

The activation current of the SMA is set to $1080 \mathrm{~mA}$ as per datasheet, and when applied to the wire it opens the clutch in approximately $6 \mathrm{~s}$, as reported in the snapshots in Fig. 4c, and then it stabilizes to an open position. Once the supply is powered off, the clutch immediately starts closing. The relaxation is slower than the opening, with approximately $30 \mathrm{~s}$ required to obtain a full closure again. For this reason, the separation of the two continuum robots takes $6 \mathrm{~s}$, whereas connecting them by opening and then closing the SMA takes approximately 36 seconds (6 opening, 30 closing, without considering the time needed to move the robots to an appropriate relative position). Thus, the preferred way to connect the two robots is by mechanically pushing the tips together to force the clutch open, rather than by using the more consuming SMA-based method. However, the latter can be used when an accurate relative positioning of the robots cannot be reached, or if the robots cannot exert enough force to open the clutch by elastically deforming the flexible hinge.

\section{EXPERIMENTAL TESTS AND RESULTS}

To evaluate the advantages of continuum robot cooperation on system stiffness improvement, the displacement of individual and cooperative continuum robots under the effect of an external load on their tip has been evaluated and compared. Furthermore, the proposed SMAbased interface mechanism has been demonstrated in a realcase scenario by having two continuum robots meet and connect within a glovebox mockup for a decommissioning operation in a nuclear facility.

\section{A. Stiffness evaluation}

To evaluate the stiffness of the continuum robot, we have studied the RAIN-snake robot [8] in various configurations. The results for three exemplary configurations are here reported: a straight configuration, the $\mathrm{C}$-shaped configuration in Fig. 5a, and the S-shaped configuration in Fig. 5b. As reported in Fig, 5c, the straight configuration deflects $25 \%$ more than the example $\mathrm{S}$-shaped configuration and $30 \%$ more of the example C-shaped configuration under the same load. Thus, a straight configuration is arguably the worst for a continuum robot with a load on the tip. 


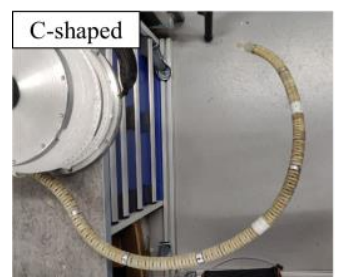

a.

c.

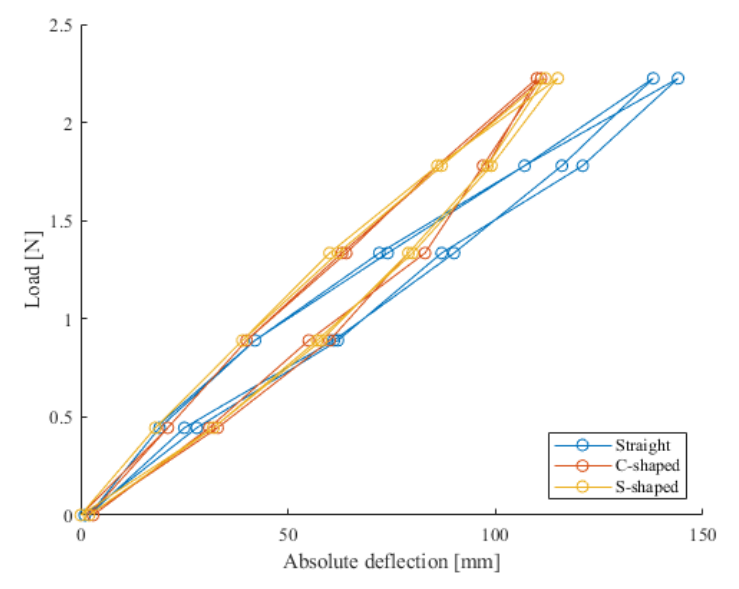

Figure 5. Stiffness evaluation of a continuum robot in various shapes: a. Cshaped configuration (first section bending right, all the other sections bending left); b. S-shaped configuration (sections 2 to 4 bending right, sections 5 to 7 bending left); c. Deflection under load of the tip of the RAINsnake continuum robot in three configurations (straight, C-shaped, S-shaped), acquired with a VICON system calibrated to an accuracy of $0.034 \mathrm{~mm}$.

When in a straight configuration, the behavior of the continuum robot is assumed to resemble the one of a cantilever beam. Thus, the maximum deflection $\delta$ of a straight continuum robot with length $l$ can be estimated as

$$
\delta=\frac{P l^{3}}{3 E I},
$$

where $P$ is the payload applied at the tip of the continuum robot, $E$ is the elastic modulus of the beam, and $I$ its equivalent bending moment of inertia. By approximating the behavior of a continuum robot to the one of a hollow cylinder with inner diameter $d_{\text {in }}$ and outer diameter $d_{\text {out }}$, it is possible to define the elastic modulus of the continuum robot in a straight configuration as

$$
E=\frac{64 P l^{3}}{3 \delta \pi\left(d_{\text {out }}{ }^{4}-d_{\text {in }}\right)^{4}} .
$$

To validate the cantilever beam assumption, the deflection under different loads of the RAIN-snake robot [8] has been studied by tracking the displacement of the backbone at the distal ends of the $4^{\text {th }}, 6^{\text {th }}$, and $8^{\text {th }}$ (tip) sections with a VICON motion capture system with four Vantage 16 cameras, calibrated to an accuracy of $0.034 \mathrm{~mm}$. The acquired values have been compared to the theoretical deflection of a cantilever beam with the same length, with a maximum error of $4.4 \%$ at the tip, $7.3 \%$ at the end of the $6^{\text {th }}$ section and $9.8 \%$ at the end of the $4^{\text {th }}$ section. Overall, the results show that the ideal cantilever model approximates well the behavior of a straight continuum robot. Thus, a stiffness coefficient $K$, defined from (2) as the ratio between $P$ and $\delta$, can be used to compare the performance of individual robots and to estimate the increase in stiffness due to conjunctive cooperation.

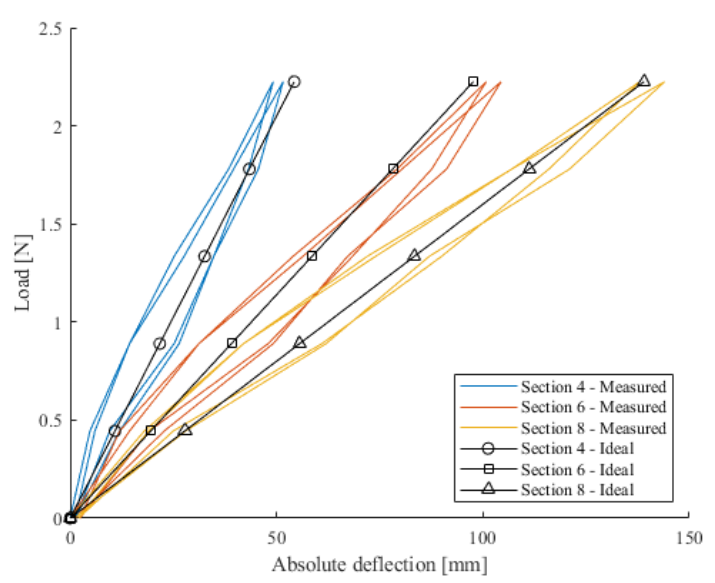

Figure 6. Deflection under load: comparison between the RAIN-snake robot and an ideal cantilever beam of equal length (hollow cylinder).

To evaluate the stiffness of the proposed robots, the displacement of two individual robot and of a cooperating system has been acquired with a VICON motion capture system with four Vantage 16 cameras, calibrated to an accuracy of $0.057 \mathrm{~mm}$. The setup is illustrated in Fig. 7a, which shows the FLARE robot [8] as robot 1 and the RAINsnake [4] as robot 2 engaging in a cantilever configuration, while the cameras capture the position of the two markers on the tip to measure deflection under load. Each experimental test characterizes the behavior of the continuum robots under five consecutive loading cycles through the following steps:

1. The continuum robots are initialized to their "straight" cantilever position with tension control. The tension of the supporting cables is set to approximately $25 \mathrm{~N}$.

2. A calibrated disk holder of $45.36 \mathrm{~g}(0.1 \mathrm{lb})$, shown in Fig. $7 b$, is installed on tip of the robot as per Figs. $6 c$ and $6 \mathrm{~d}$, and the resulting deflection is measured.

3. Up to four calibrated disks of $45.36 \mathrm{~g}$ each $(0.1 \mathrm{lb})$, shown in Fig. 7b, are progressively added to the disk holder at the tip of the robot, measuring its motion and recording the deflection after each added disk.

4. The system is progressively unloaded by removing the disks one by one and then the disk holder. After each unloading step, the deflection is recorded.

5. Steps 2 to 4 are repeated another four times to characterize the behavior of the continuum robot(s) over a total of 5 loading-unloading cycles.

The test has been repeated in three different configurations to measure the deflection of the FLARE robot, of the RAINSnake robot, and of the two robots connected by the proposed SMA-based mechanism, with the results reported in Fig. 8.

In terms of absolute deflection (Fig. 8a), the worst behavior is observed in the FLARE robot: its large deflection (up to 132 $\mathrm{mm}$ at maximum load) is characterized by a large hysteresis (up to $21 \mathrm{~mm}$ ) between loading and unloading, as well as a displacement between the starting points of consecutive cycles, with consecutive unloaded states characterized by a progressively greater deflection. In particular, the first cycle is characterized by a large deflection of $67 \mathrm{~mm}$ that is never recovered after unloading, while the permanent deflection is 
smaller (less than $8 \mathrm{~mm}$ ) for subsequent cycles. This displacement is likely caused by a loss in tension in the actuation cables after loading, as the FLARE robot is not designed to support tip loads. The RAIN-Snake robot shows a slightly larger absolute deflection (up to $139 \mathrm{~mm}$ ) and a similar hysteresis $(25 \mathrm{~mm})$, but a smaller starting displacement $(25$ $\mathrm{mm}$ in the first cycle, $2 \mathrm{~mm}$ or less in subsequent ones), showing its capability to reliably manipulate a load of $2.2 \mathrm{~N}$.

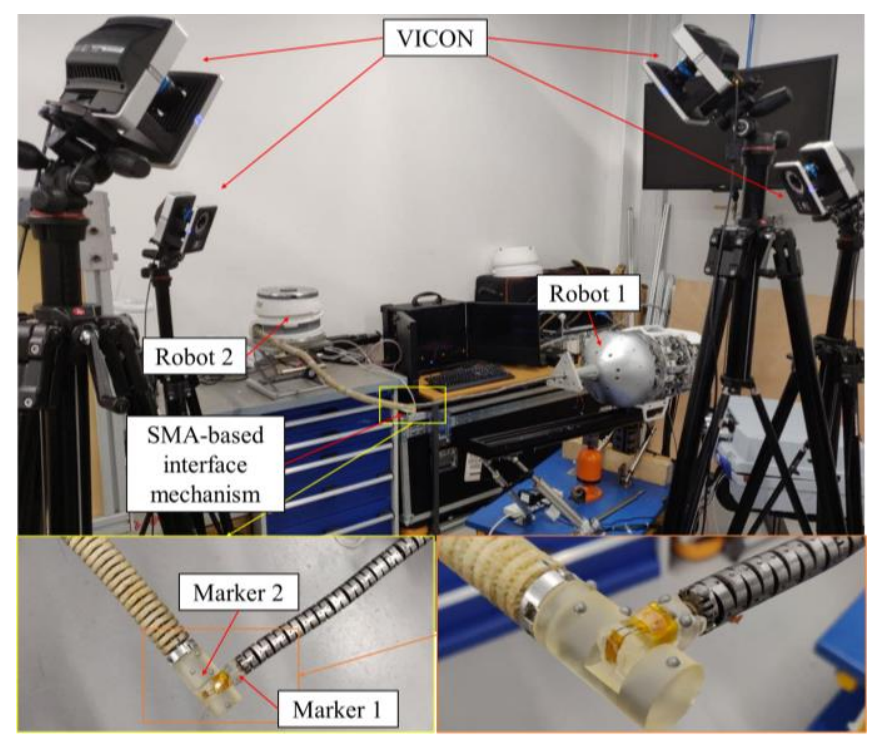

a.

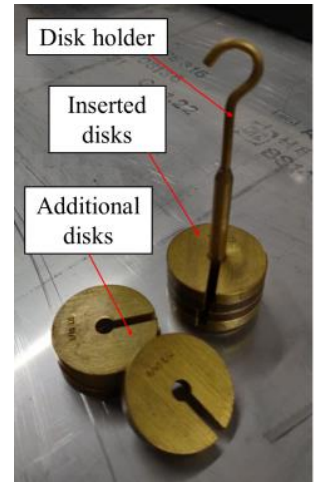

b.

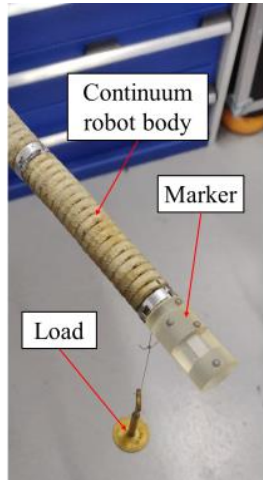

c.

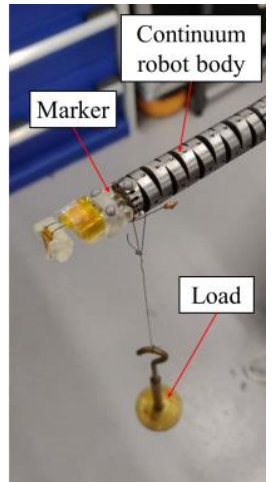

d.
Figure 7. Experimental evaluation of stiffness through deflection under a known load: a. Setup with two continuum robots equipped with the proposed SMA-based interface mechanism and markers for the VICON motion capture system; b. Calibrated weight set to load/unload the robots; c. RAIN-Snake continuum robot with load; d: FLARE robot continuum with load.

However, these two robots are characterized by different geometries, and a more significative comparison is reported in Fig. $8 \mathrm{~b}$ with the deflection normalized over robot length. The normalized values further highlight the better performance of RAIN-Snake over FLARE, with comparable deflections at maximum load but different displacements $(0.2 \%$ for RAINSnake, $1.1 \%$ for FLARE in cycles 2 to 5). The performance of the cooperating system has been normalized over a geometrical average of the lengths of the constituting robots, and shows a maximum normalized deflection of $5.1 \%$, compared to $14.0 \%$ for RAIN-Snake and $22.2 \%$ for FLARE. These results can be further filtering by removing the transient behavior of the first cycle to analyze the average deflection of the robot over cycles 2 to 5, as shown in Fig. 8c. In this graph, the behavior of the individual robots is comparable, with RAIN-Snake showing a greater deflection and FLARE being hindered by the permanent displacement of its unloaded configuration. A significant increase in performance can be appreciated on the average loading-unloading curve of the cooperating system, which reduces both maximum deflection and hysteresis by approximately $70 \%$ and shows how the support of the RAIN-Snake compensates for the displacement between consecutive unloaded configurations in FLARE.

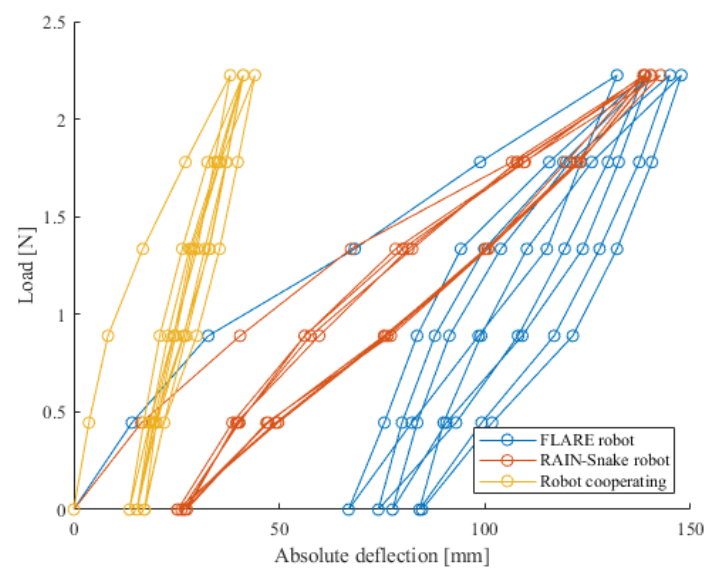

a.

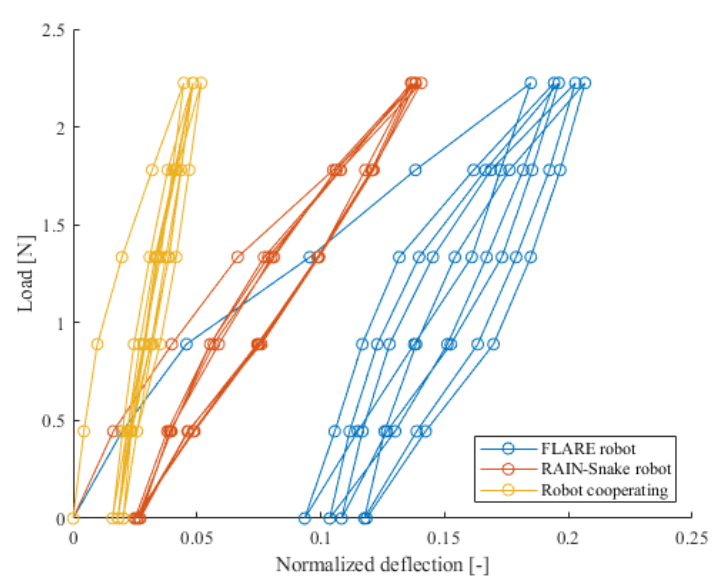

b.

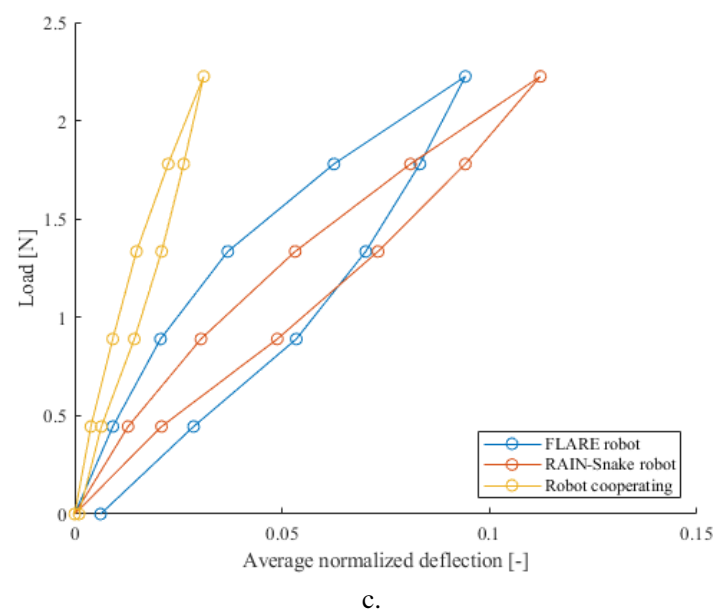

Figure 8. Experimental results: a. Absolute value of the deflection measured as displacement of the marker along the vertical (gravity) axis; $b$. Deflection of the robot normalized by the continuum robot's length; c. Average of the normalized deflection from testing cycle 2 to 5 . 
To better compare the increase in stiffness, a stiffness coefficient $K$ can be extracted from the linear regression of the data in Fig. 8a, which gives an estimation of the ratio between payload $P$ and deflection $\delta$. As shown in Table I, cooperation results in a significant performance improvement. Thus, these results validate the hypothesis of improved static behavior for conjunctive cooperation between continuum robots and characterize it with a numerical example. The whole cooperating system performs better than the sum of its parts, with an increase in stiffness coefficient $K$ greater than $200 \%$ the stiffness coefficient of the continuum robots that constitute the system, a reduction of approximately $70 \%$ in hysteresis and deflection over repeated loading/unloading cycles, and with a continuum robot compensating for the permanent deformation of the other due to cable tension loss.

TABLE I

DEFLECTION TEST PARAMETERS AND RESULTS

\begin{tabular}{cccc}
\hline \hline Parameter & RAIN-Snake & FLARE & $\begin{array}{c}\text { Cooperating } \\
\text { System }\end{array}$ \\
\hline Length & $1015 \mathrm{~mm}$ & $715 \mathrm{~mm}$ & - \\
\hline Outer diameter & $20 \mathrm{~mm}$ & $12 \mathrm{~mm}$ & - \\
\hline Inner diameter & $8 \mathrm{~mm}$ & $6 \mathrm{~mm}$ & - \\
\hline Maximum & $25 \mathrm{~mm}$ & $21 \mathrm{~mm}$ & $6 \mathrm{~mm}$ \\
hysteresis & $2.5 \%$ & $2.9 \%$ & $0.7 \%$ \\
\hline Maximum & $139 \mathrm{~mm}$ & $132 \mathrm{~mm}$ & $38 \mathrm{~mm}$ \\
deflection & $13.7 \%$ & $18.5 \%$ & $4.5 \%$ \\
\hline Displacement & $25 \mathrm{~mm}$ & $67 \mathrm{~mm}$ & $13 \mathrm{~mm}$ \\
on cycle 1 & $2.5 \%$ & $9.4 \%$ & $1.5 \%$ \\
\hline Max. displ. on & $2 \mathrm{~mm}$ & $8 \mathrm{~mm}$ & $2 \mathrm{~mm}$ \\
cycles 2+ & $0.2 \%$ & $1.1 \%$ & $0.2 \%$ \\
\hline Stiffness coeff. & $0.0147 \mathrm{~N} / \mathrm{mm}$ & $0.0112 \mathrm{~N} / \mathrm{mm}$ & $0.0437 \mathrm{~N} / \mathrm{mm}$ \\
\hline \hline
\end{tabular}

\section{B. Glovebox demonstration}

The decommissioning of nuclear gloveboxes requires the inspection and manipulation of the objects contained therein, currently performed by human operators exposed to the hazards of a radioactive environment. Continuum robots can automate the inspection of these items [4], and the proposed cooperation strategies would enable retrieval without relying on other robots or human operators. Therefore, the proposed system has been demonstrated within a glovebox mock-up, with the two continuum robots accessing the environment from two separate access ports (Fig. 9a), meeting in the workspace (Fig. 9b), and then connecting to each other. Once joint, the robots cooperate by lifting a weight (Fig. 9c) and then release the clutch by heating the SMA (Fig. 9d). The chosen payload of $2.67 \mathrm{~N}$ would be impossible for the individual robots to lift. In fact, the RAIN-Snake can lift up to $2 \mathrm{~N}$, while FLARE is designed to lift its end-effector but no additional loads. When a heavier load is imposed on the tip on the individual robots, any attempt to lift it results in backbone deflection rather than tip motion, as illustrated in Fig. 9e for RAIN-Snake trying to lift $2.67 \mathrm{~N}$. Lifting this excessive weight can also cause a mechanical failure in tendons or joints. Conversely, the cooperative system can lift this payload, as in Fig. 9f, thanks to the increase in mechanical performance. With this test, the capability of the proposed system to operate within a nuclear application is shown. Furthermore, the results highlight how a cooperating system can outperform individual continuum robots by performing tasks with a load higher than the combined payload of the constituting robots.

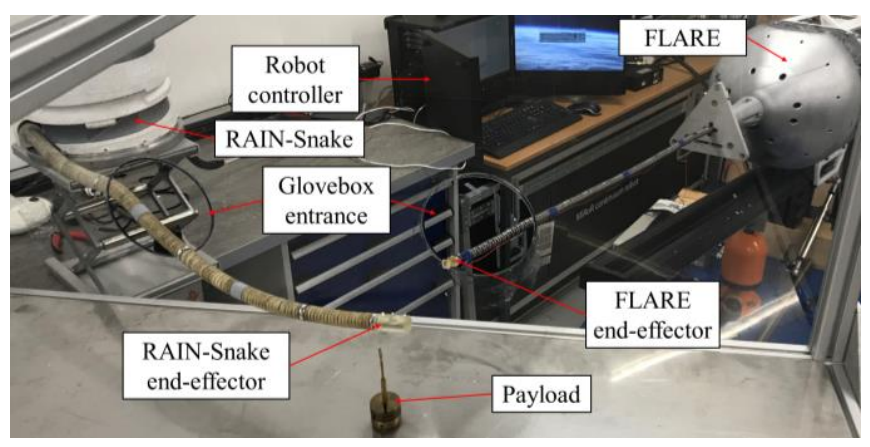

a.
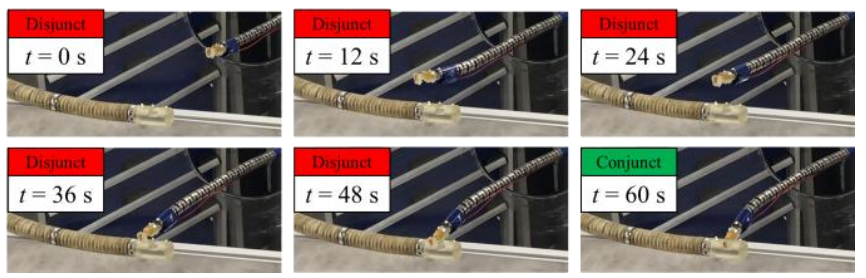

b.
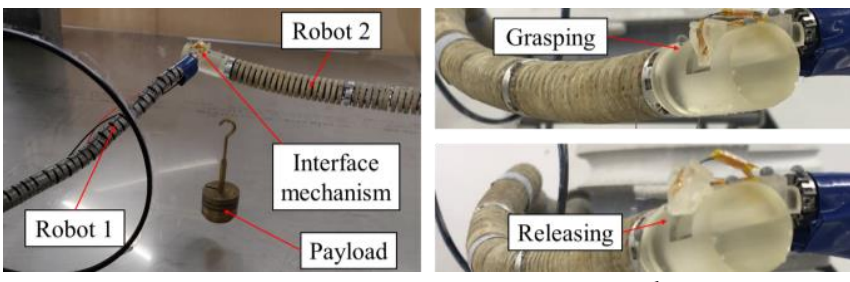

c.

d.

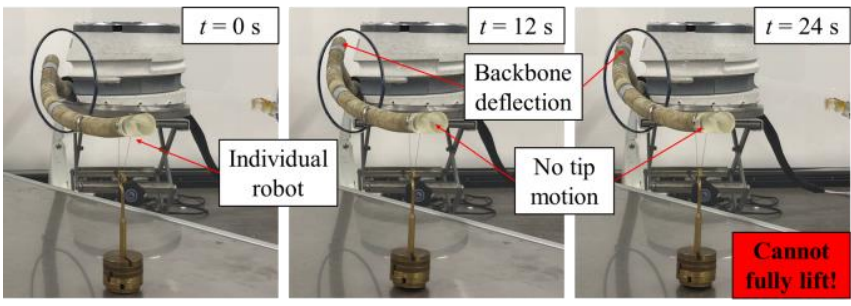

e.
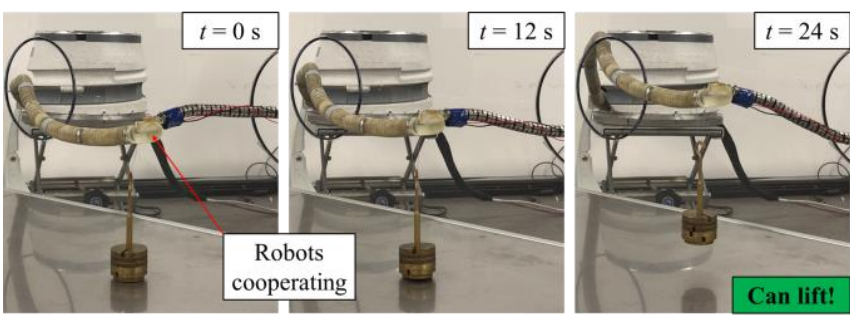

Figure 9. Demonstration in a nuclear glovebox mock-up: a. Experimental setup; b. The two robots navigating and connecting together; c. Cooperative weight lifting; d. Clutch release after operation; e. An individual robot trying to lift a load heavier than its payload; $f$. The cooperating system lifting 2.67 $\mathrm{N}$, more than the combined payload of the constituting robots.

\section{CONCLUSION}

In this paper, conjunctive (i.e., with physical contact) cooperation is proposed as a solution to significantly improve the performance of continuum robots and overcome their intrinsically low stiffness. This cooperation capability can be added to any existing continuum robot, as shown in the experimental examples of this paper, rather than requiring a custom-made design with a shared actuation as in the limited 
cooperation achieved by previous studies. The main results of this research can be summarized in the following points:

- Analysis of continuum robot cooperation: Cooperating tasks are classified according to the interaction between the robots and their reliance on each other, and the relative requirements are identified.

- A novel SMA-based mechanical interface: The proposed design enables force transmission between continuum robots, allowing them to act and navigate independently, physically connect before operation, and fully release the engagement after the task. An example prototype can perform the engagement immediately thanks to a mechanical snap fit, support up to $10 \mathrm{~N}$ of external load when closed, and release the grasp in $6 \mathrm{~s}$ by heating a shape-memory alloy.

- Performance evaluation of cooperating continuum robots: The static performance of cooperating continuum robots is evaluated in terms of stiffness. A stiffness coefficient $K$, defined as the ratio between payload $P$ and the corresponding tip deflection $\delta$, is proposed as performance index. The experiments show a significant improvement during cooperation (more than 200\% stiffness increase and a reduction of $70 \%$ of the robots' deflection under load).

- Demonstration in a nuclear application: Cooperation is demonstrated in a retrieval operation for glovebox decommissioning. In the test, the robots navigate, connect, and then lift a payload higher than the combined payloads of the individual continuum robots that constitute the system.

In conclusion, the cooperation between continuum robots here introduced is a unique way to overcome the physical limits of these robots, which can be used to significantly improve their mechanical performance not exclusively for newly designed continuum robots, but also by adapting existing ones with customized end-effectors. This work focuses on the mechanical aspect of the cooperation, but opens several interesting topics for the community, such as combined shape optimization for maximum stiffness increase. Future areas for development include modeling, redundancy management and robot synchronization and coordination.

\section{REFERENCES}

[1] I.D. Walker, H. Choset, and G.S. Chirikjian. "Snake-like and continuum robots." In Springer handbook of robotics, pp. 481-498. Springer, Cham, 2016.

[2] M. Russo, L. Raimondi, X. Dong, D. Axinte, and J. Kell, "Taskoriented optimal dimensional synthesis of robotic manipulators with limited mobility." Robot Comput Integr Manuf 69 (2021):102096.

[3] J. Burgner-Kahrs, D.C. Rucker, and H. Choset, "Continuum robots for medical applications: A survey." IEEE Trans. Robot. 31, no. 6 (2015): 1261-1280.

[4] A. Mohammad, M. Russo, Y. Fang, X. Dong, D. Axinte, J. Kell, “An efficient follow-the-leader strategy for continuum robot navigation and coiling." IEEE Robot. Autom. Lett. 6, no. 4 (2021): 7493-7500.

[5] K. Oliver-Butler, J. Till, and C. Rucker. "Continuum robot stiffness under external loads and prescribed tendon displacements." IEEE Trans. Robot. 35, no. 2 (2019): 403-419.
[6] G. Smoljkic, D. Reynaerts, J. Vander Sloten and E. Vander Poorten, "Compliance computation for continuum types of robots", Proceedings IEEE/RSJ International Conference on Intelligent Robots and Systems (IROS), Chicago, IL, pp. 1066-1073, 2014.

[7] D. Ji, T.H. Kang, S. Shim, and J. Hong. "Analysis of twist deformation in wire-driven continuum surgical robot." International Journal of Control, Automation and Systems 18, no. 1 (2020): 10-20.

[8] M. Wang, X. Dong, W. Ba, A. Mohammad, D. Axinte, and A. Norton. "Design, modelling and validation of a novel extra slender continuum robot for in-situ inspection and repair in aeroengine." Robot Comput Integr Manuf 67 (2021): 102054.

[9] D. Alatorre, B. Nasser, A. Rabani, A. Nagy-Sochacki, X. Dong, D. Axinte, and J. Kell. "Teleoperated, in situ repair of an aeroengine: Overcoming the internet latency hurdle." IEEE Robot Autom Mag 26, no. 1 (2018): 10-20.

[10] M. Wang, D. Palmer, X. Dong, D. Alatorre, D. Axinte, and A. Norton. "Design and development of a slender dual-structure continuum robot for in-situ aeroengine repair." In 2018 International Conference on Intelligent Robots and Systems (IROS), pp. 5648-5653. IEEE, 2018.

[11] X. Dong, M. Wang, A. Mohammad, M. Russo, W. Ba, A. Norton, J. Kell, D. Axinte. "Continuum robots collaborate for safe manipulation of high-temperature flame to enable repairs in extreme environments." IEEE/ASME Transactions on Mechatronics (2021). In press.

[12] C.B. Black, J. Till, and D.C. Rucker. "Parallel continuum robots: Modeling, analysis, and actuation-based force sensing." IEEE Transactions on Robotics 34, no. 1 (2017): 29-47.

[13] S. Lilge, K. Nülle, G. Böttcher, S. Spindeldreier, and J. Burgner-Kahrs. "Tendon actuated continuous structures in planar parallel robots: A Kinematic analysis." Jnl. of Mechanisms and Robotics (2020): 1-34.

[14] B. Mauzé, R. Dahmouche, G.J. Laurent, A.N. André, P. Rougeot, P. Sandoz, and C. Clévy. "Nanometer precision with a planar parallel continuum robot." IEEE Robot. Autom. Lett. 5, no. 3 (2020): 38063813.

[15] K. Nuelle, T. Sterneck, S. Lilge, D. Xiong, J. Burgner-Kahrs, and T. Ortmaier. "Modeling, calibration, and evaluation of a tendon-actuated planar parallel continuum robot." IEEE Robot. Autom. Lett. 5, no. 4 (2020): 5811-5818.

[16] A.W. Mahoney, P.L. Anderson, P.J. Swaney, F. Maldonado, and R.J. Webster. "Reconfigurable parallel continuum robots for incisionless surgery." In 2016 IEEE/RSJ International Conference on Intelligent Robots and Systems (IROS), pp. 4330-4336. IEEE, 2016.

[17] A.L. Orekhov, C.B. Black, J. Till, S. Chung, and D.C. Rucker. "Analysis and validation of a teleoperated surgical parallel continuum manipulator." IEEE Robot. Autom. Lett. 1, no. 2 (2016): 828-835.

[18] G. Böttcher, S. Lilge and J. Burgner-Kahrs, "Design of a reconfigurable parallel continuum robot with tendon-actuated kinematic chains." IEEE Robot. Autom. Lett. 6, no. 2 (2021): 1272-1279.

[19] P.L. Anderson, A.W. Mahoney and R.J. Webster. Continuum reconfigurable parallel robots for surgery: shape sensing and state estimation with uncertainty. IEEE Robot. Autom. Lett. 2, no. 3 (2017): $1617-1624$.

[20] N. Rahman, N.J. Deaton, J. Sheng, S.S. Cheng, and J.P. Desai, "Modular FBG bending sensor for continuum neurosurgical robot." IEEE Robot. Autom. Lett. 4, no. 2 (2019): 1424-1430.

[21] E.W. Hawkes, L.H. Blumenschein, J.D. Greer, and A.M. Okamura, "A soft robot that navigates its environment through growth", Science Robotics, Vol. 2, Issue 8, pp. 1-7, 2017.

[22] J. Gallentine, M.B. Wooten, M. Thielen, I.D. Walker, T. Speck, and K.J. Niklas, "Searching and intertwining: Climbing plants and GrowBots", Frontiers Robotics and AI, Vol. 7, Article 118.

[23] J.P. Merlet. Parallel robots. Vol. 128. Springer Science \& Business Media, 2005.

[24] C. Armanini, I. Hussain, M. Z. Iqbal, D. Gan, D. Prattichizzo and F. Renda, "Discrete Cosserat Approach for Closed-Chain Soft Robots: Application to the Fin-Ray Finger," IEEE Transactions on Robotics (2021), doi: 10.1109/TRO.2021.3075643. 